\title{
Spot the Red Herring: Breastfeeding, Fruitpurée, and Infant Autonomic Functioning-The Generation R Study
}

\author{
BRAM DIERCKX, ANNE THARNER, JOKE H.M. TULEN, VINCENT W. JADDOE, ALBERT HOFMAN, FRANK C. VERHULST, \\ AND HENNING TIEMEIER
}

\begin{abstract}
The Generation R Study Group [B.D., V.W.J.], Departments of Child and Adolescent Psychiatry [A.T., F.C.V., H.T.], Psychiatry [J.H.M.T.], and Epidemiology [A.H.], Erasmus MC University Medical Center, Rotterdam, Zuid-Holland, 3000 CA, The Netherlands
\end{abstract}

\begin{abstract}
Several studies have suggested that breastfeeding is related to infant autonomic functioning. The authors investigated whether this is a causal relation. In all, 444 mothers reported breastfeeding practices 2 mo postpartum. Infant autonomic functioning was assessed by heart rate variability at age $14 \mathrm{mo}$, after discontinuation of breastfeeding. The dose-dependent association between breastfeeding and infant autonomic functioning was tested with linear regression models adjusted for multiple confounders. The authors investigated the relation of fruitpurée consumption with infant autonomic functioning. Fruitpurée consumption has similar socioeconomic epiphenomena but is not related via the same causal mechanism to autonomic regulation as breastfeeding. Nonbreastfed infants had high sympathetic modulation [7.87 $\log \left(\mathrm{ms}^{2}\right) / \mathrm{SD}, 95 \% \mathrm{CI}$ : 7.71-8.02], partially breastfed infants had intermediate sympathetic modulation [7.75 $\left.\log \left(\mathrm{ms}^{2}\right) / \mathrm{SD}, 95 \% \mathrm{CI}: 7.51-7.82\right]$, sympathetic modulation of exclusively breastfed infants was low [7.63 $\log \left(\mathrm{ms}^{2}\right) /$ SD, 95\% CI: 7.50-7.77]. However, this association could be explained by socioeconomic confounders. Furthermore, fruitpurée consumption was similarly associated with reduced infant sympathetic modulation. The association between breastfeeding practices and infant sympathetic modulation was accounted for by socioeconomic and environmental factors. We found a similar association between fruitpurée consumption and autonomic functioning, further suggesting that the association between breastfeeding and infant autonomic functioning is noncausal. (Pediatr Res 70: 417-422, 2011)
\end{abstract}

$\mathrm{B}$ reastfeeding may have several beneficial effects on cardiovascular functioning, including decreased adiposity and blood pressure in later life $(1,2)$. Similarly, studies suggest that breastfeeding is related to lower total plasma cholesterol and less intima-media thickness in adults $(3,4)$. Research also indicated that breastfeeding has a beneficial effect on the infant's autonomic cardiovascular regulation. Two decades ago, di Pietro et al. (5) studied autonomic functioning and observed lower heart rate and higher heart rate variability in breastfed neonates compared with formula-fed neonates. Similar findings were reported in infants aged 1 and 4 mo.

Received October 12, 2010; accepted April 22, 2011.

Correspondence: Henning Tiemeier, M.D., Ph.D., Erasmus MC, Department of Child and Adolescent Psychiatry, P.O. Box 2060, 3000 CB Rotterdam, The Netherlands; e-mail: h.tiemeier@erasmusmc.nl

Supported by the Erasmus Medical Center Rotterdam, the Erasmus University Rotterdam, and the Netherlands Organisation for Health Research and Development (ZonMW), by Unilever S.A. for nutritional research in the Generation R study with a grant, and by an independent research grant of the European Community's 7th Framework Programme (FP7/2008-2013) under grant agreement no. 212652 (NUTRIMENTHE Project "The Effect of Diet on the Mental Performance of Children").
Breastfed infants had a lower resting heart rate than infants who were formula fed (6). Long-term benefits of such an autonomic profile have been described; in adults, it was shown that lower resting heart rates and higher heart rate variability are associated with a lower risk of cardiovascular mortality $(7,8)$.

Different aspects of breastfeeding could account for the effect on the infant's cardiovascular regulation. First, research in adults showed that the intake of long-chain polyunsaturated fatty acids is associated with increased vagal modulation and a decreased sympathetic/vagal ratio (9). The composition of human milk, namely the mixture of fatty acids, meets the nutritional needs of the human infant best (10). Although formula milk is currently prepared with various concentrations and mixtures of long-chain poly-unsaturated fatty acids, plasma levels of these fatty acids in formula-fed infants do not reach those found in breastfed infants $(11,12)$.

Second, breastfeeding may influence infant cardiovascular regulation because it generates less physiological stress during feeding than bottle feeding. Research in preterm infants has shown that breastfed infant's heart rate, respiratory rate, and body temperature increase more during feeding than their bottle fed counterparts $(13,14)$.

Third, as Feldman and Eidelman (15) showed, in preterm infants skin-to-skin contact promotes infant autonomic maturation. They documented that infants treated with kangaroo care, which emphasizes mother-infant skin-to-skin contact, showed a greater increase in vagal tone than infants who received no such care.

However, the evidence for differential autonomic functioning in breastfed infants compared with formula fed infants is not conclusive. The existing studies measured infant autonomic functioning during the breastfeeding period only. Hence, it is unclear whether the effect reported persists after mothers discontinued breastfeeding. Moreover, these studies were relatively small, making them particularly vulnerable to biases.

Further, there are clear socioeconomic differences between mothers who chose to breastfeed and those who chose to bottle feed (16). Studies without adequate control for confounders may erroneously infer a causal effect of breastfeeding (17). The US Agency of Healthcare Research and Quality reviewed the current literature on health benefits of breastfeeding and concluded that although breastfeeding is associated with a reduced risk of many

Abbreviations: HF, high frequency; LF, low frequency 
A

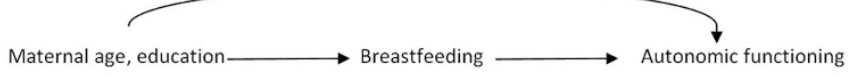

B

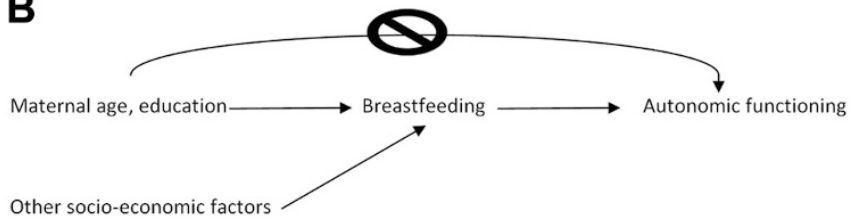

Figure 1. (A) Confounding by socioeconomic factors. (B) Residual confounding after controlling for selected socioeconomic factors.

diseases in infants, most of the associations were detected in observational studies. They warn not to infer causality based on such findings because of the likelihood of confounding (18).

Indeed, previous studies only controlled for sex, age, and weight $(5,6)$. Other environmental factors may also account for a spurious association between breastfeeding and infant autonomic functioning. Mothers, who breastfeed, display greater sensitivity in interactions with their infants (19). Maternal sensitivity, in particular, is associated with infant autonomic functioning as measured by increased vagal tone (20).

The presence of confounding can be represented by diagrams, known as directed acyclic graphs, as illustrated for birth defects by Hernán et al. $(21,22)$. These graphs depict variables, linked by arrows which represent causal effects. Figure $1 A$ is such a graph and shows that socioeconomic factors influence both breastfeeding and autonomic functioning. Socioeconomic factors violate the assumption of ignorability; they act as a confounder and need to be controlled for. Socioeconomic and educational factors are very important determinants of breastfeeding $(23,24)$. Hence, as also shown in Figure 1A, whether breastfeeding is causally related to infant autonomic functioning, it is a potential mediator between socioeconomic factors and infant autonomic functioning. However, as shown in Figure $1 B$, even with diligent control for known confounders, residual confounding by socioeconomic factors remains a challenge in observational research of breastfeeding (25).

An alternative approach to controlling for confounders is to compare the strength of the association between breastfeeding and infant autonomic functioning with another feeding parameter, which is related to similar socioeconomic and behavioral epiphenomena as breastfeeding, but which lacks the specific biological properties of breastfeeding that may influence infant autonomic functioning (26).

In a large, well-defined, population-based cohort, we assessed the effect of breastfeeding practices and the effect of fruitpuree consumption on autonomic functioning at $14 \mathrm{mo}$. We hypothesized that breastfeeding has a direct biological effect on infant autonomic functioning, and that the association with autonomic functioning is stronger than the association of a comparator without the postulated physiological effects.

\section{METHODS}

Participants. This study was conducted within the Focus Cohort of the Generation R Study, a population-based prospective cohort study from fetal life until young adulthood $(27,28)$. Measurements of autonomic indices were added to the protocol of the examination round, while assessment was already ongoing. Physiological measurements were available for 528 infants. Our study population consisted of the 444 children, for whom both data on breastfeeding practices and autonomic indices were available. Written informed consent was obtained from all participants. Approval for the study was granted by the Medical Ethics Committee of the Erasmus Medical Center, Rotterdam.

Breastfeeding. Information on breastfeeding practices at 2 mo of age was collected by postal questionnaire. Mothers were asked to indicate whether they breastfed their infants exclusively, whether they formula fed their infants exclusively, or whether they combined both breastfeeding and formula feeding.

Psychophysiologic measurements. Mothers and their infants were invited to our laboratory 14 mo after birth. Trained research assistants performed the physiological measurements. We registered heart rate using a precordial, three pole ECG lead, sampled at $512 \mathrm{~Hz}$. Furthermore, we monitored the breathing pattern using a piezo-electric transducer. Signals were recorded using a Vitaport 3 digital recorder (Temec Inc., Kerkrade, The Netherlands). We recorded for $8 \mathrm{~min}$, while the infant was at ease in its mother's lap. To help the infant relax, we played an episode of the Teletubbies $\odot$ (BBC/Ragdoll Limited). Recordings did not start until signals had reached a stabilized steady state.

Power spectral analysis. We analyzed the recorded data using custom made software. Because irregular, slow breathing distributes the variation attributable to vagal modulation across the frequency spectrum, we manually selected 100 to $180 \mathrm{~s}$ of the ECG where breathing was most regular. R-top detection was conducted on the selected data window. Interbeat intervals were examined on the basis of the time between consecutive R-tops in the ECG. We performed spectral analysis using discrete Fourier transformation, based on nonequidistant sampling of the R-wave indices (29). The low frequency (LF) component of heart rate variability, defined between 0.04 and $0.14 \mathrm{~Hz}$, is dependent on sympathetic modulation and certainly in older individuals partly on vagal modulation as well. However, vagal modulation of heart rate is dependent on respiratory frequency, which is much higher in infants, likely attenuating the vagal component of LF variation. Hence, for reasons of simplicity, we will refer to LF variation as a measure of sympathetic modulation. Furthermore, we adjusted the upper bound of the high frequency (HF) band, which is dependent solely on vagal modulation, according to recommendations from literature, to allow for the infant's higher respiratory frequency. We defined our HF band between 0.15 and $1.04 \mathrm{~Hz}$ (30).

Covariates. Gender of the infant, GA at birth, and weight at time of assessment were used as covariates because they influence mean heart rate and heart rate variability. We obtained GA at birth and birth weight from community midwife and hospital registries. To address confounding by socioeconomic factors, information on maternal age, maternal parity, maternal age at first birth, and maternal level of highest completed education was available as well as information on paternal level of highest completed education and household income (16). Information on socioeconomic factors was obtained by a questionnaire completed by the mother and her partner. Confounding by maternal sensitivity and confidence in caretaking was addressed by using the confidence-in-caretaking scale of the Mother and Baby Scale, which was part of the postal questionnaires sent to mothers when their child was 2 mo old. The scale contains 13 items, which use a six-point Likert scale. Covariates were assumed to be missing at random and were imputed by the mean.

Statistical analysis. For the nonresponse analysis, we compared the 444 mothers and children for whom breastfeeding data were available with the 84 mothers and children who were not included in the analysis because of missing data on breastfeeding. To describe the study participants, we compared the characteristics of the infants who received no breastfeeding $(n=$ 156) to the characteristics of the infants who received partial breastfeeding $(n=72)$ and the infants who received exclusive breastfeeding $(n=216)$. We also investigated the association between the infant's fruitpurée consumption and the family's socioeconomic status. For continuous variables, we used ANOVA and for dichotomous variables $\chi^{2}$ tests. We conducted a power analysis with $\mathrm{G}^{*}$ power to determine the detectable effect size (31). The physiological variables LF and HF were log transformed to achieve a normal distribution. In addition, we divided the physiological variables by their own $\mathrm{SD}$ to facilitate interpretation of the estimates.

We introduced sets of variables into successive analysis of covariance (ANCOVA) models to investigate whether the association between breastfeeding and infant autonomic functioning attenuated when confounders were controlled for. Additionally, we tested a dose-response effect with linear regression. Breastfeeding was entered as an ordinal variable with a value of 0 , 1 , or 2 . We used partial breastfeeding as the intermediate category and calculated a $p$ trend. 
First, we studied the univariate relation between breastfeeding and infant autonomic functioning, termed model 1 . In model 2, we adjusted for basic confounders comprised of infant gender, infant birth weight, and infant weight at 14 mo. Finally, in model 3, we adjusted for socioeconomic factors and maternal sensitivity.

However, model 3 may not give the definite answer to the study question. Even in large, well-defined studies, which rigorously control for socioeconomic and other factors, it is virtually impossible to rule out residual confounding (25). Breastfeeding may be a marker for other factors related to infant autonomic functioning that were not fully captured by the covariables we used, e.g. differences in rearing style or maternal stress during and after pregnancy (32).

To address this issue, we contrasted the relation between breastfeeding and infant autonomic functioning with the relation between another infant feeding parameter and infant autonomic functioning. We chose infant fruitpurée consumption as the best available contrasting variable. Generally, it is the earliest addition to the infant's diet, complimentary to continued breast or bottle feeding. Fruitpurée, similar to breastfeeding, is seen as healthy nourishment for infants.

On the basis of our data, we know that fruitpurée consumption is related to similar environmental epiphenomena as breastfeeding. Fruitpurée lacks the long-chain unsaturated fatty acids found in breastmilk, it lacks breastfeeding's specific physiologic process, and it is not implicitly associated with skin-toskin contact. In short, it has none of the characteristics that could underlie the putative association between breastfeeding and infant autonomic functioning. Analogous to the analyses of breastfeeding, we performed linear regressions to determine the association between fruitpurée consumption and infant autonomic functioning. Statistical analyses were performed with the Statistical Package for the Social Sciences 15.0 (SPSS Inc., Chicago, IL).

Comparator-Fruitpurée consumption. Fruitpurée consumption was assessed at 12 months after birth using a postal questionnaire. Fruitpurée is typically fed from 6 mo onwards only. However, a nutritional comparator from the first 6 mo of life is not conceivable because many infants are exclusively breastfed in this period. Fruitpurée consumption was assessed on a seven-point scale but collapsed to three categories, because of small numbers and to enable comparison with breastfeeding: 1-less than one portion of fruitpurée per day, 2-one portion of fruitpurée a day, and 3 - more than one portion of fruitpurée a day.

Nonresponse and power analysis. Children without information on breastfeeding had similar autonomic functioning, as well as similar weight at birth and at $14 \mathrm{mo}$. Similarly, their mothers did not differ on age or education (data not shown). Power analysis demonstrated that our study could detect small effects $(f=0.15)$ at 0.85 power and 0.85 and small to medium effects $(f=$ 0.19 ) at 0.95 power, with $\alpha=0.05$.

\section{RESULTS}

Forty-six percent of infants were exclusively breastfed at 2 mo of age, whereas $16 \%$ were partly breastfed. The remainder, $38 \%$, received no breastfeeding at 2 mo. Table 1 summarizes the baseline characteristics for infants according to these categories. Infants, who received no breastfeeding, had a similar boy/girl ratio, birth weight, and weight at 14 mo compared with those who received partial or exclusive breastfeeding. Furthermore, infants who received no breastfeeding consumed roughly similar amounts of fruitpurée as children receiving partial or exclusive breastfeeding $\left(\chi^{2}=4.6, p=0.3\right)$.

Breastfeeding was positively associated with maternal education $\left(\chi^{2}=43.6, p<0.001\right)$, paternal education $\left(\chi^{2}=29.6\right.$, $p<0.001)$, and maternal age at first child birth $(F=3.3, p=$ $0.04)$ but was not associated with current maternal age $(F=$ 2.1, $p=0.1)$ or household income $\left(\chi^{2}=4.4, p=0.1\right)$.

Infants' fruitpurée consumption at 12 mo was positively associated with maternal education $\left(\chi^{2}=16.1, p=0.03\right)$, as well as current maternal age $(F=2.995, p=0.05)$, maternal age at first childbirth $(F=4.28, p=0.01)$, and household income $\left(\chi^{2}=7.17, p=0.03\right)$ but was not associated with paternal education $\left(\chi^{2}=4.7, p=0.3\right)$, maternal parity $(F=$ $0.51, p=0.6$ ), or with household income.

First, we explored the association between breastfeeding and infant HF power, a measure of vagal modulation of the heart rate. The unadjusted model showed that there was no relation between infant breastfeeding and vagal modulation $(p$ for trend $=0.70)$. We found similar levels of vagal modulation in nonbreastfed infants $\left[6.97 \mathrm{log}\left(\mathrm{ms}^{2}\right) / \mathrm{SD}, 95 \% \mathrm{CI}\right.$ : 6.81-7.13], partially breastfed [7.02 $\log \left(\mathrm{ms}^{2}\right) / \mathrm{SD}, 95 \% \mathrm{CI}$ : $6.78-7.26]$ and fully breastfed infants $\left[6.86 \log \left(\mathrm{ms}^{2}\right) / \mathrm{SD}\right.$, 95\% CI: 6.72-7.00]. Adding covariates to subsequent models yielded similar results (data not shown).

Table 2 depicts the results of successive regression models for the association between breastfeeding and LF power, a measure of infant sympathetic modulation. The unadjusted model is pictured in Figure 2. Nonbreastfed infants had high sympathetic modulation [7.87 $\log \left(\mathrm{ms}^{2}\right) / \mathrm{SD}, 95 \% \mathrm{CI}: 7.71-$ 8.02], sympathetic modulation of partially breastfed infants was intermediate [7.75 $\left.\log \left(\mathrm{ms}^{2}\right) / \mathrm{SD}, 95 \% \mathrm{CI}: 7.51-7.98\right]$ whereas sympathetic modulation of exclusively breastfed infants was lowest [7.63 $\log \left(\mathrm{ms}^{2}\right) / \mathrm{SD}, 95 \% \mathrm{CI}$ : 7.50-7.77]. This means that sympathetic modulation in exclusively breastfed infants was 0.23 of an SD lower than in infants who

Table 1. Characteristics of the study population

\begin{tabular}{|c|c|c|c|}
\hline Focus cohort & $\begin{array}{l}\text { No breastfeeding (reference group), } \\
\qquad N=156\end{array}$ & $\begin{array}{l}\text { Partial breastfeeding, } \\
\qquad N=72\end{array}$ & $\begin{array}{l}\text { Exclusive breastfeeding, } \\
\qquad N=216\end{array}$ \\
\hline \multicolumn{4}{|l|}{ Parental indices } \\
\hline Age mother at intake, y (SD) & $31.4(4.2)$ & $31.9(3.7)$ & $32.2(3.3)$ \\
\hline \multicolumn{4}{|l|}{ Maternal education } \\
\hline Secondary education or lower $(\%)$ & 53.9 & 32.4 & 23.1 \\
\hline Higher education, phase $1(\%)$ & 23.7 & 31.0 & 26.4 \\
\hline Higher education, phase $2(\%)$ & 22.4 & 36.6 & 50.5 \\
\hline \multicolumn{4}{|l|}{ Infant indices } \\
\hline Female $(\%)$ & 49 & 49 & 49 \\
\hline Birth weight in $g$ (SD) & $3479(575)$ & $3474(595)$ & $3590(541)$ \\
\hline Weight at 14 mo $g(S D)$ & $10,579(1082)$ & $10,484(1033)$ & $10,486(1022)$ \\
\hline \multicolumn{4}{|l|}{ Fruitpurée consumption } \\
\hline Less than one a d (\%) & 13.4 & 8.3 & 7.4 \\
\hline One a d $(\%)$ & 80.9 & 86.1 & 84.7 \\
\hline More than one a d (\%) & 5.7 & 5.6 & 7.9 \\
\hline Vagal activity, $\log \mathrm{ms}^{2}(\mathrm{SD})$ & $2.88(0.42)$ & $2.90(0.44)$ & $2.83(0.42)$ \\
\hline Sympathetic modulation, $\log \mathrm{ms}^{2}(\mathrm{SD})$ & $2.79(0.34)$ & $2.75(0.35)$ & $2.71(0.38)$ \\
\hline
\end{tabular}


Table 2. Amount of breastfeeding received at age 2 mo and infant sympathetic modulation

\begin{tabular}{|c|c|c|c|c|c|c|}
\hline \multirow[b]{2}{*}{$n=444$} & \multirow{2}{*}{$\begin{array}{c}\text { No breastfeeding } \\
\text { Mean } \log \mathrm{ms}^{2} / \mathrm{SD} \\
\text { reference }\end{array}$} & \multicolumn{2}{|c|}{ Partial breastfeeding } & \multicolumn{2}{|c|}{ Exclusive breastfeeding } & \multirow[b]{2}{*}{$\begin{array}{c}\text { Dose-response } \\
p \text { for trend }\end{array}$} \\
\hline & & $\begin{array}{c}\text { Mean log } \\
\mathrm{ms}^{2} / \mathrm{SD}\end{array}$ & $\begin{array}{l}\text { Difference with } \\
\text { reference }(95 \% \mathrm{CI})\end{array}$ & $\begin{array}{c}\text { Mean log } \\
\mathrm{ms}^{2} / \mathrm{SD}\end{array}$ & $\begin{array}{l}\text { Difference with } \\
\text { reference }(95 \% \mathrm{CI})\end{array}$ & \\
\hline Model 1 & 7.87 & 7.75 & $-0.12(-0.40$ to 0.16$)$ & 7.63 & $-0.23(-0.44 \text { to }-0.02)^{*}$ & 0.03 \\
\hline Model 2 & 7.86 & 7.74 & $-0.12(-0.40$ to 0.17$)$ & 7.64 & $-0.22(-0.42 \text { to }-0.01)^{*}$ & 0.04 \\
\hline Model 3 & 7.86 & 7.75 & $-0.11(-0.40$ to 0.17$)$ & 7.64 & $-0.22(-0.45$ to 0.01$)$ & 0.12 \\
\hline
\end{tabular}

Results displayed are from successive ANCOVA models. Model 1, unadjusted; model 2, as model 1, additionally adjusted for infant gender, infant birth weight, and infant weight at 14 mo. Model 3 as model 2, additionally adjusted for maternal age, parental education, household income, maternal parity, and maternal age at first birth, as well as maternal confidence in caretaking. Dose-response effects were investigated with linear regressions.

* Different from reference group at $p<0.05$.

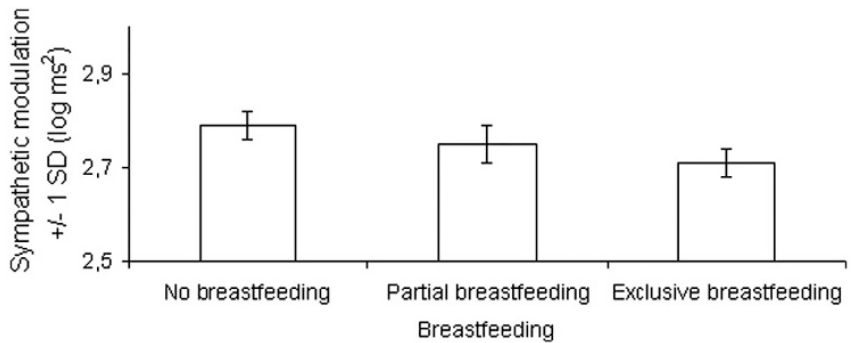

Figure 2. Breastfeeding and infant sympathetic modulation. Based on an unadjusted linear regression model.

received no breastfeeding. Linear regression analysis showed a dose response effect for breastfeeding.

Next, we examined whether we could identify factors that explained the association between breastfeeding and infant sympathetic functioning. Infant gender and weight characteristics did not change the model substantially (Table 2). In contrast, the introduction of socioeconomic factors and maternal sensitivity attenuated the relation between breastfeeding and infant sympathetic modulation. When these confounders were taken in account, nonbreastfed infants had similar sympathetic modulation [7.84 log $\left(\mathrm{ms}^{2}\right) / \mathrm{SD}, 95 \%$ CI: 7.67-8.00] compared with partially breastfed infants $\left[7.75 \log \left(\mathrm{ms}^{2}\right) / \mathrm{SD}\right.$, 95\% CI: 7.52-7.98] and compared with exclusively breastfed infants [7.65 $\log \left(\mathrm{ms}^{2}\right) / \mathrm{SD}, 95 \%$ CI: 7.50-7.79]. A dose response effect was no longer present.

Table 3 displays the results of successive linear regression models for the association between fruitpuree and our measure of infant sympathetic modulation, LF power. The unadjusted linear regression model showed that the more fruitpuree an infant consumed, the lower the infants' sympathetic modulation, as depicted in Figure 3. Children eating less than one portion of fruitpuree each day had the highest sympathetic modulation [7.99 $\left.\log \left(\mathrm{ms}^{2}\right) / \mathrm{SD}, 95 \% \mathrm{CI}: 7.70-8.28\right]$, followed by children eating on average one portion of fruitpurée a day [7.69 $\log \left(\mathrm{ms}^{2}\right) / \mathrm{SD}, 95 \%$ CI: 7.60-7.79], whereas those who ate more than one portion of fruitpurée a day had the lowest sympathetic modulation $\left[7.38 \log \left(\mathrm{ms}^{2}\right) / \mathrm{SD}, 95 \% \mathrm{CI}\right.$ : 7.10-7.71]. Even in a fully adjusted model fruitpurée consumption was associated with lower infant sympathetic modulation, probably indicating residual confounding.

\section{DISCUSSION}

Infants, who were exclusively breastfed at 2 mo of age, had lower sympathetic modulation than infants not breastfed.
However, the observed relation could be accounted for by socioeconomic and educational factors. Higher infant fruitpurée consumption was associated with lower infant sympathetic modulation. No association between breastfeeding practices and infant vagal modulation was found.

Before we discuss these findings, some methodological comments have to be made. Our study benefits from a large sample size and a prospective design. Furthermore, we tried to address confounding by socioeconomic and educational factors by controlling for several indicators. The Focus Cohort is ethnically homogeneous to exclude potential confounding or effect modification by ethnicity. At the same time, this limits the generalizability of the results to other ethnic groups.

Earlier studies reported associations between breastfeeding and infant autonomic functioning $(5,6)$. However, these studies were small and did not control for socioeconomic and educational factors $(16,19)$.

The association between breastfeeding and infant autonomic functioning disappeared after correction for socioeconomic factors. However, the actual degree of attenuation of the association between breastfeeding and autonomic functioning achieved by correcting for socioeconomic factors was minor, suggesting little effect of control. In addition, adding covariates to a model does not necessarily improve its' validity and hence the causal inferences made on the basis of the model. For example, research has shown that breastfeeding influences maternal sensitivity toward her infant (19). Maternal sensitivity might also be influenced by infant autonomic functioning, because hypothetically autonomic functioning influences the level of interaction an infant displays. In that case, controlling for maternal sensitivity would introduce bias to the model.

However, as shown in Figure $1 B$, even in studies which rigorously control for socioeconomic and other factors, it is virtually impossible to rule out residual confounding. Parenting and attachment styles, as well the infant's diet in addition breastfeeding and fruitpurée are examples of potential residual confounders, which we were not able to address in this study.

Presence of residual confounding can lead to erroneously dismissal of the mediating effect of breastfeeding for socioeconomic factors and autonomic functioning (33). This would mean that our model without adjustment for confounders cannot be completely discounted.

Literature recommends several approaches to study causal inferences (26). A randomized controlled trial is seen as the golden standard. However, the established benefits of breast- 
Table 3. Amount of fruitpurée consumed at age 14 mo and infant sympathetic modulation

\begin{tabular}{|c|c|c|c|c|c|c|}
\hline \multirow[b]{3}{*}{$n=444$} & \multicolumn{6}{|c|}{ Fruitpurée consumption } \\
\hline & \multirow{2}{*}{$\begin{array}{c}<1 \text { portion/d } \\
\text { Mean } \log \mathrm{ms}^{2} / \mathrm{SD} \\
\text { reference }\end{array}$} & \multicolumn{2}{|r|}{1 portion/d } & \multicolumn{2}{|r|}{$>1$ portion/d } & \multirow[b]{2}{*}{$\begin{array}{c}\text { Dose-response } \\
P \text { for trend }\end{array}$} \\
\hline & & $\begin{array}{l}\text { Mean log } \\
\mathrm{ms}^{2} / \mathrm{SD}\end{array}$ & $\begin{array}{l}\text { Difference with } \\
\text { reference }(95 \% \mathrm{CI})\end{array}$ & $\begin{array}{l}\text { Mean log } \\
\mathrm{ms}^{2} / \mathrm{SD}\end{array}$ & $\begin{array}{l}\text { Difference with } \\
\text { reference }(95 \% \mathrm{CI})\end{array}$ & \\
\hline Model 1 & 7.99 & 7.69 & $-0.30(-0.61$ to 0.01$)$ & 7.38 & $-0.62(-1.05 \text { to }-0.18)^{*}$ & 0.007 \\
\hline Model 2 & 8.02 & 7.69 & $-0.32(-0.63 \text { to }-0.02)^{*}$ & 7.35 & $-0.67(-1.10 \text { to }-0.23)^{*}$ & 0.008 \\
\hline Model 3 & 8.02 & 7.69 & $-0.33(-0.63 \text { to }-0.02)^{*}$ & 7.36 & $-0.66(-1.10 \text { to }-0.21)^{*}$ & 0.008 \\
\hline
\end{tabular}

Results displayed are from successive ANCOVA models. Model 1, unadjusted; model 2, as model 1, additionally adjusted for infant gender, infant birth weight, and infant weight at 14 mo. Model 3 as model 2, additionally adjusted for maternal age, parental education, household income, maternal parity, and maternal age at first birth, as well as maternal confidence in caretaking and household income. Dose-response effects were investigated with linear regressions.

* Different from reference group at $p<0.05$.

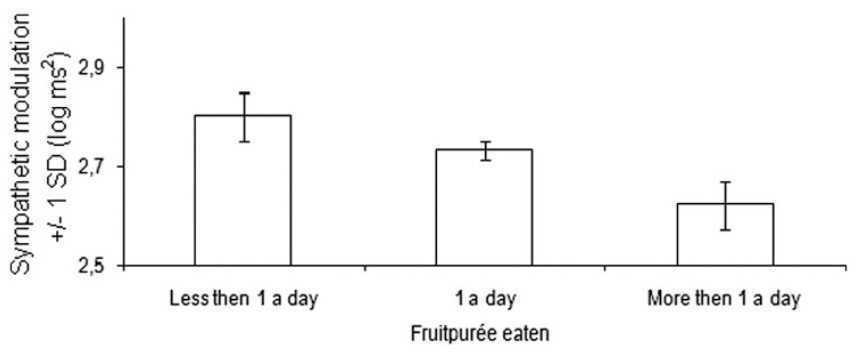

Figure 3. Fruitpurée and infant sympathetic modulation. Based on an unadjusted linear regression model.

feeding make randomly withholding breastfeeding from infants unethical. Some research has used the randomized assignment of mothers to a breastfeeding promotion program (34). Because of the limited influence of breastfeeding promotion programs on breastfeeding practice in a general population setting in the West, the number of participants needed to successfully detect differences in autonomic functioning make this approach unfeasible for our purposes (35).

Another approach is to use an instrumental variable, which is related to the exposure variable, in this case breastfeeding, but not to confounding factors or to the outcome, in this case infant autonomic functioning. Such a variable can be genetic, as in Mendelian randomization, or environmental (26). Unfortunately, to our knowledge, no instrumental variable, genetic, or otherwise, applicable in breastfeeding research has been identified.

A third approach, is to compare the effect of breastfeeding with the effect of a contrasting variable. A contrasting variable should be an indicator of similar feeding or health-related parameters but lacks breastfeeding's proposed causal properties. Thus, if there is indeed a causal relation, the effect of breastfeeding on infant autonomic functioning should be larger. This approach has been used in the study of intrauterine exposure, e.g. where it is possible, to compare the associations of prenatal maternal smoking and prenatal paternal smoking with infant outcome (26).

Reverse causality also represents a problem. It is conceivable that calmer infants, which are easier to breastfeed (36), already have different autonomic functioning, e.g. lower sympathetic modulation. This would then account for the relation between breast feeding and sympathetic modulation we observed. Unfortunately, ruling out reverse causality calls for multiple measurements of autonomic functioning and multiple measurements of the infant's agreeableness to be breastfed, which few studies provide.

In our study, frequent infant fruitpurée consumption is the contrasting variable. It is related to the similar environmental epiphenomena as breastfeeding, including maternal socioeconomic status, education, and caretaking as well as maternal concepts about health and nourishment. There was no significant association between infant fruitpurée consumption and breastfeeding, thus breastfeeding and fruitpurée consumption did not identify the same mother-infant pairs. Infant fruitpurée does not contain fatty acids, some of which, the long-chain poly-unsaturated fatty acids, have been associated with autonomic functioning. Finally, fruitpurée consumption does not come with skin-to-skin contact. Nonetheless, our data show that, similar to breastfeeding, infants who eat more fruitpurée have lower sympathetic modulation. This supports the notion that the association between breastfeeding and infant autonomic functioning may be similarly noncausal but instead the result of residual confounding. Although, strictly speaking, we cannot rule out that the relation of breastfeeding and fruitpurée consumption to autonomic functioning is explained via different biological mechanisms. If a common component of the infant's diet, like fruitpurée, is similarly associated with autonomic functioning as breastfeeding but lacks it properties, this certainly casts doubt on the specificity of the postulated mechanisms and the relevance of breastfeeding for low infant autonomic arousal.

Finally, although our study shows no lasting effect of breast feeding on infant autonomic functioning, we cannot rule out a transient effect, limited to the breast feeding period itself. However, it is unlikely that such a transient effect would have substantial future health benefits.

In conclusion, our study does not support a causal relation between breastfeeding and infant autonomic functioning. Instead, it underlines the problem of confounding in observational studies of breastfeeding. Further, we presented a novel way to address the dilemma of confounding and overcorrection in research concerning breastfeeding. It can help to compare the impact of breastfeeding to the impact of another feeding parameter later in life that is not causally related to outcome of interest.

Acknowledgments. The Generation R Study is conducted by the Erasmus Medical Center Rotterdamin close collaboration with the Faculty of Social Sciences of the Erasmus 
University Rotterdam, the Municipal Health Service Rotterdam area, the Rotterdam Homecare Foundation, and the Stichting Trombosedienst \& Artsenlaboratorium Rijnmond (STAR), Rotterdam. We thank the contribution of general practitioners, hospitals, midwives, and pharmacies in Rotterdam.

\section{REFERENCES}

1. Buyken AE, Karaolis-Danckert N, Remer T, Bolzenius K, Landsberg B, Kroke A 2008 Effects of breastfeeding on trajectories of body fat and BMI throughout childhood. Obesity (Silver Spring) 16:389-395

2. Lawlor DA, Smith GD 2005 Early life determinants of adult blood pressure. Curr Opin Nephrol Hypertens 14:259-264

3. Marmot MG, Page CM, Atkins E, Douglas JW 1980 Effect of breast-feeding on plasma cholesterol and weight in young adults. J Epidemiol Community Health 34:164-167

4. Martin RM, Ebrahim S, Griffin M, Davey Smith G, Nicolaides AN, Georgiou N, Watson S, Frankel S, Holly JM, Gunnell D 2005 Breastfeeding and atherosclerosis: intima-media thickness and plaques at 65-year follow-up of the Boyd Orr cohort. Arterioscler Thromb Vasc Biol 25:1482-1488

5. di Pietro JA, Larson SK, Porges SW 1987 Behavioral and heart rate pattern differences between breast-fed and bottle-fed neonates. Dev Psychol 23:467-474

6. Butte NF, Smith EO, Garza C 1991 Heart rates of breast-fed and formula-fed infants J Pediatr Gastroenterol Nutr 13:391-396

7. Kannel WB, Kannel C, Paffenbarger RS Jr, Cupples LA 1987 Heart rate and cardiovascular mortality: the Framingham Study. Am Heart J 113:1489-1494

8. Tsuji H, Larson MG, Venditti FJ Jr, Manders ES, Evans JC, Feldman CL, Levy D 1996 Impact of reduced heart rate variability on risk for cardiac events. The Framingham Heart Study. Circulation 94:2850-2855

9. Mozaffarian D, Stein PK, Prineas RJ, Siscovick DS 2008 Dietary fish and omega-3 fatty acid consumption and heart rate variability in US adults. Circulation 117:11301137

10. World Health Organization 2003 Global Strategy for Infant and Young Child Feeding. World Health Organization, Geneva. Available at: http://www.who.int/ nutrition/publications/gs_infant_feeding_text_eng.pdf. Accessed June 1, 2011.

11. Chirouze V, Lapillonne A, Putet G, Salle BL 1994 Red blood cell fatty acid composition in low-birth-weight infants fed either human milk or formula during the first months of life. Acta Paediatr Suppl 405:70-77

12. Koletzko B, Lien E, Agostoni C, Bohles H, Campoy C, Cetin I, Decsi T, Dudenhausen JW, Dupont C, Forsyth S, Hoesli I, Holzgreve W, Lapillonne A, Putet G, Secher NJ, Symonds M, Szajewska H, Willatts P, Uauy R 2008 The roles of long-chain polyunsaturated fatty acids in pregnancy, lactation and infancy: review of current knowledge and consensus recommendations. J Perinat Med 36:5-14

13. Chen CH, Wang TM, Chang HM, Chi CS 2000 The effect of breast- and bottlefeeding on oxygen saturation and body temperature in preterm infants. J Hum Lact 16:21-27

14. Meier P, Anderson GC 1987 Responses of small preterm infants to bottle- and breast-feeding. MCN Am J Matern Child Nurs 12:97-105

15. Feldman R, Eidelman AI 2003 Skin-to-skin contact (Kangaroo Care) accelerates autonomic and neurobehavioural maturation in preterm infants. Dev Med Child Neurol 45:274-281

16. Simmons D 1997 NIDDM and breastfeeding. Lancet 350:157-158

17. Der G, Batty GD, Deary IJ 2006 Effect of breast feeding on intelligence in children: prospective study, sibling pairs analysis, and meta-analysis. BMJ 333:945
18. Ip S, Chung M, Raman G, Trikalinos TA, Lau J 2009 A summary of the Agency for Healthcare Research and Quality's evidence report on breastfeeding in developed countries. Breastfeed Med 4:S17-S30

19. Britton JR, Britton HL, Gronwaldt V 2006 Breastfeeding, sensitivity, and attachment. Pediatrics 118:e1436-e1443

20. Kaplan LA, Evans L, Monk C 2008 Effects of mothers' prenatal psychiatric status and postnatal caregiving on infant biobehavioral regulation: can prenatal programming be modified? Early Hum Dev 84:249-256

21. Hernán MA, Hernández-Díaz S, Werler MM, Mitchell AA 2002 Causal knowledge as a prerequisite for confounding evaluation: an application to birth defects epidemiology. Am J Epidemiol 155:176-184

22. Pearl J 2000 Causality: Models, Reasoning, and Inference. Cambridge University Press, Cambridge

23. Yngve A, Sjostrom M 2001 Breastfeeding in countries of the European Union and EFTA: current and proposed recommendations, rationale, prevalence, duration and trends. Public Health Nutr 4:631-645

24. van Rossem L, Oenema A, Steegers EA, Moll HA, Jaddoe VW, Hofman A, Mackenbach JP, Raat H 2009 Are starting and continuing breastfeeding related to educational background? The generation R study. Pediatrics 123:e1017-e1027

25. Martin RM, Goodall SH, Gunnell D, Davey Smith G 2007 Breast feeding in infancy and social mobility: 60-year follow-up of the Boyd Orr cohort. Arch Dis Child 92:317-321

26. Smith GD 2008 Assessing intrauterine influences on offspring health outcomes: can epidemiological studies yield robust findings? Basic Clin Pharmacol Toxicol 102:245-256

27. Jaddoe VW, Mackenbach JP, Moll HA, Steegers EA, Tiemeier H, Verhulst FC, Witteman JC, Hofman A 2006 The Generation R Study: design and cohort profile. Eur J Epidemiol 21:475-484

28. Jaddoe VW, Bakker R, van Duijn CM, van der Heijden AJ, Lindemans J, Mackenbach JP, Moll HA, Steegers EA, Tiemeier H, Uitterlinden AG, Verhulst FC, Hofman A 2007 The Generation R Study Biobank: a resource for epidemiological studies in children and their parents. Eur J Epidemiol 22:917-923

29. van Steenis HG, Tulen JH, Mulder LJ 1994 Heart rate variability spectra based on non-equidistant sampling: the spectrum of counts and the instantaneous heart rate spectrum. Med Eng Phys 16:355-362

30. Bar-Haim Y, Marshall PJ, Fox NA 2000 Developmental changes in heart period and high-frequency heart period variability from 4 months to 4 years of age. Dev Psychobiol 37:44-56

31. Faul F, Erdfelder E, Lang AG, Buchner A 2007 G*Power 3: a flexible statistical power analysis program for the social, behavioral, and biomedical sciences. Behav Res Methods 39:175-191

32. Dierckx B, Tulen JH, van den Berg MP, Tharner A, Jaddoe VW, Moll HA, Hofman A, Verhulst FC, Tiemeier H 2009 Maternal psychopathology influences infant heart rate variability: Generation R Study. Psychosom Med 71:313-321

33. Herting JR 2002 Evaluating and rejecting true mediation models: a cautionary note. Prev Sci 3:285-289

34. Kramer MS, Aboud F, Mironova E, Vanilovich I, Platt RW, Matush L, Igumnov S, Fombonne E, Bogdanovich N, Ducruet T, Collet JP, Chalmers B, Hodnett E, Davidovsky S, Skugarevsky O, Trofimovich O, Kozlova L, Shapiro S 2008 Breastfeeding and child cognitive development: new evidence from a large randomized trial. Arch Gen Psychiatry 65:578-584

35. Chung M, Raman G, Trikalinos T, Lau J, Ip S 2008 Interventions in primary care to promote breastfeeding: an evidence review for the U.S. Preventive Services Task Force. Ann Intern Med 149:565-582

36. Niegel S, Ystrom E, Hagtvet KA, Vollrath ME 2008 Difficult temperament, breastfeeding, and their mutual prospective effects: the Norwegian Mother and Child Cohort Study. J Dev Behav Pediatr 29:458-462 